\title{
ON THE SPECTRUM OF THE SCHUR COMPLEMENT OF THE STOKES OPERATOR VIA CONFORMAL MAPPING *
}

\author{
SÁNDOR ZSUPPÁN ${ }^{\dagger}$
}

\begin{abstract}
We investigate the eigenvalues and eigenfunctions of the Schur complement operator of the first kind Stokes problem posed on a plane domain and give results on the number, multiplicity and stability of these eigenvalues in dependence on the problem domain.
\end{abstract}

1. Introduction. The Schur complement operator of the Stokes problem on a plane domain $\Omega$,

$$
\begin{aligned}
-\Delta u+\operatorname{grad} p & =f, \quad \operatorname{div} u=0 \text { on } \Omega, \\
u & =0 \text { on } \partial \Omega,
\end{aligned}
$$

is defined by $\mathcal{S}=\operatorname{div} \Delta_{0}^{-1}$ grad, where $\Delta_{0}$ denotes the vector Laplace operator corresponding to homogeneous Dirichlet boundary values. The eigenvalues of the Schur complement operator, i.e.

$$
\Delta u=\operatorname{grad} p ; \operatorname{div} u=\lambda p \text { in } \Omega ; \text { and } u=0 \text { on } \partial \Omega,
$$

are important for stability and error estimates connected with the Stokes problem since here the so-called inf-sup constant

$$
\inf _{0 \neq p \in L_{2,0}} \sup _{0 \neq u \in\left(H_{0}^{1}\right)^{2}} \frac{(\operatorname{div} u, p)_{0}^{2}}{(u, u)_{1}(p, p)_{0}}=\beta_{0}^{2}>0
$$

is involved, and also for the iterative solution of discretized Stokes and Navier-Stokes problems. Here is $L_{2,0}$ the subspace of $L_{2}(\Omega)$ of square integrable functions with zero integral over $\Omega$, where $(p, q)_{0}=\int_{\Omega} p q d x$ is the scalar product. $\left(H_{0}^{1}\right)^{2}$ is the Sobolev space of vector functions with componentwise generalized derivatives in $L_{2}(\Omega)$ and with zero boundary values in the sense of traces on the boundary of $\Omega$, where the scalar product is defined by $(u, v)_{1}=\int_{\Omega} \sum_{i, j=1}^{2} \frac{\partial u_{i}}{\partial x_{j}} \frac{\partial v_{i}}{\partial x_{j}} d x$. Preliminary work on different properties of related eigenvalue problems has been done by [1]-[5], [7], [8], [10], [18][20]. However, explicit values of the inf-sup constant for special domains are known only in a few cases: for the circle, the annulus [3], and the ellipse, see [10], and for an infinite strip - assuming periodicity along the strip [13], and, in the three-dimensional case, for the sphere [18]. Some lower and upper bounds for inf-sup constants of several domains are derived in [16], the use of this knowledge for the acceleration of iterative methods is shown in [17]. [6] contains related work.

We start in Section 2 from results of [20] (see Lemma 1 and Theorem 2 below) and examine in detail the resulting eigenvalue problem which turns out to be connected to an eigenvalue problem for a matrix $\mathcal{M}_{S}$. This gives Corollary 9 below for the eigenvalues of the Schur complement operator on plane domains arising as conformal maps of the unit disc by a univalent polynomial function. So we have the opportunity to describe the eigenfunctions and eigenvalues and to calculate the inf-sup constant of such domains. In Section 4 we generalize Theorem 2. We give examples how this

\footnotetext{
* Received May 16, 2003; accepted for publication August 30, 2004.

†Berzsenyi Dániel Evangélikus Gimnázium, Széchenyi tér 11, H-9400 Sopron, Hungary (zsuppan@emk.nyme.hu).
} 
theorem can be used to calculate the eigenvalues of the Schur complement of the Stokes operator (and hence the inf-sup constant) on a plane domain if we have the conformal map of the unit disc onto that domain. In Theorem 19 we investigate the relationship between the multiplicity of the eigenvalues of the Schur complement operator and the symmetry of the domain. We show in Section 5 the continuous dependence of the matrix $\mathcal{M}_{S}$ on the mapping function. We also investigate some examples concerning criteria of continuity for the inf-sup constant with respect to the domain.

2. Domains obtainable by polynomial mappings. The following result gives the representation for the solutions of (1) - physically the momentum equation - in the homogeneous case and for simply connected plane domains $\Omega$ which are conformal maps of the unit disc $D$.

Lemma 1. (Lemma 3.7 in [20]) Let $g$ be the conformal map of the unit disc $D$ onto $\Omega, p=2 \operatorname{Re} f, f$ holomorphic on $D$. The functions $u: D \rightarrow \mathbb{C}$, for which the transformed function $u \circ g^{-1}$ fulfils the homogeneous momentum equation

$$
\Delta\left(u \circ g^{-1}\right)=\operatorname{grad}\left(p \circ g^{-1}\right)
$$

on $\Omega$ can be represented by the formula

$$
u=\frac{1}{2} g \bar{f}+v_{1}+\bar{v}_{2}
$$

with holomorphic functions $v_{1}$ and $v_{2}$. Further $u$ has the divergence

$$
\operatorname{div}\left(u \circ g^{-1}\right) \circ g=\operatorname{Ref}+2 \operatorname{Re} \frac{v_{1}^{\prime}}{g^{\prime}}
$$

Using the representation (5) and (6), Theorem 3.1 in [20] offers a possibility to describe the eigenvalues and eigenfunctions of the Schur complement operator $\mathcal{S}$ of the Stokes equation on plane domains which are polynomial conformal maps of the unit disc $D$.

TheOrem 2. (Theorem 3.1 in [20]) Let $g$ be a bijective conformal mapping $D \rightarrow \Omega$ of the form

$$
g(z)=\sum_{m=0}^{M} a_{m} z^{m}
$$

with $g^{\prime} \neq 0$ on $\bar{D}$,

$$
\frac{1}{g^{\prime}(z)}=\sum_{\ell=0}^{\infty} b_{\ell} z^{\ell} .
$$

Then we have for the Schur complement operator $\mathcal{S}$, defined by $\mathcal{S} p=$ divu, of the Stokes equation

- for $f(z)=z^{n}$ with $n \geq M$, the functions $p_{R}:=2 \operatorname{Ref}$ and $p_{I}:=2 \operatorname{Im} f$ are eigenfunctions of $\mathcal{S}$ to the eigenvalue $\frac{1}{2}$; 
- for $f(z)=\frac{\sum_{n=0}^{M-1} p_{n} z^{n}}{g^{\prime}(z)}$, the function $p_{R}:=2$ Ref leads to

$$
\mathcal{S} p_{R}=\frac{1}{2} p_{R}+2 \operatorname{Re} \frac{\sum_{n=0}^{M-1} q_{n} z^{n}}{g^{\prime}(z)}
$$

and $p_{I}:=2 \operatorname{Im} f$ leads to

$$
\mathcal{S} p_{I}=\frac{1}{2} p_{I}-2 \operatorname{Im} \frac{\sum_{n=0}^{M-1} q_{n} z^{n}}{g^{\prime}(z)}
$$

where the conjugate linear mapping $\left(p_{\ell}\right)_{\ell=0 \ldots M-1} \mapsto\left(q_{k}\right)_{k=0 \ldots M-1}$

$$
q_{k}=\sum_{l=0}^{M-1} s_{k, \ell} \bar{p}_{\ell}
$$

is defined by the coefficients

$$
s_{k, \ell}=\left\{\begin{array}{cr}
-\frac{k+1}{2} \sum_{m=k+1+\ell}^{M} a_{m} \bar{b}_{m-k-1-\ell} & \text { for } 0 \leq k+\ell \leq M-1 \\
0 & \text { otherwise. }
\end{array}\right.
$$

Observe that the conjugate linear mapping (11) is described by a matrix of special triangular form

$$
\mathcal{M}_{S}=-\frac{1}{2}\left(\begin{array}{cccccc}
s_{0} & s_{1} & s_{2} & \ldots & \ldots & s_{M-1} \\
2 s_{1} & 2 s_{2} & \ldots & \ldots & 2 s_{M-1} & 0 \\
3 s_{2} & \ldots & \ldots & 3 s_{M-1} & 0 & 0 \\
\vdots & & & \vdots & \vdots & \vdots \\
\vdots & & & \vdots & \vdots & \vdots \\
M s_{M-1} & 0 & 0 & \ldots & \ldots & 0
\end{array}\right)
$$

The entries of this matrix,

$$
s_{k}=a_{k+1} \bar{b}_{0}+a_{k+2} \bar{b}_{1}+\cdots+a_{M} \bar{b}_{M-k-1}
$$

for $k=0,1, \ldots, M-1$ correspond to (12).

Introduce the conjugacy operator $\mathcal{C}: x+i y \mapsto x-i y$ and the vectors $q:=$ $\left(q_{0}, \ldots, q_{M-1}\right)^{T}$ and $p:=\left(p_{0}, \ldots, p_{M-1}\right)^{T}$ using the coefficients of (11). Solving the eigenvalue problem

$$
\mathcal{M}_{S} \mathcal{C} p=\mu p,(\mu \in \mathbb{C}, p \neq 0)
$$

we have $q=\mu p$ and

$$
\frac{\sum_{n=0}^{M-1} q_{n} z^{n}}{g^{\prime}(z)}=\mu \frac{\sum_{n=0}^{M-1} p_{n} z^{n}}{g^{\prime}(z)}=\mu f(z) .
$$

Now using (9) and (10), there follows

$$
\begin{aligned}
\mathcal{S} p_{R} & =\frac{1}{2} p_{R}+2 \operatorname{Re}(\mu f(z)), \\
\mathcal{S} p_{I} & =\frac{1}{2} p_{I}-2 \operatorname{Im}(\mu f(z)) .
\end{aligned}
$$


In the following section we investigate the validity of $|\mu| \leq \frac{1}{2}$ in (15) and the case of complex eigenvalues. If $\mu$ is real and $|\mu| \leq \frac{1}{2}$ then we have

$$
\begin{aligned}
& \mathcal{S} p_{R}=\left(\frac{1}{2}+\mu\right) p_{R}, \text { with } p_{R}=2 \operatorname{Re} \frac{\sum_{n=0}^{M-1} p_{n} z^{n}}{g^{\prime}(z)} \\
& \mathcal{S} p_{I}=\left(\frac{1}{2}-\mu\right) p_{I}, \text { with } p_{I}=2 \operatorname{Im} \frac{\sum_{n=0}^{M-1} p_{n} z^{n}}{g^{\prime}(z)}
\end{aligned}
$$

where $p=\left(p_{0}, p_{1}, \ldots, p_{M-1}\right)^{T}$ is an eigenvector to the eigenvalue $\mu$.

Therefore the second part of Theorem 2 implies that $\lambda=\frac{1}{2}+\mu$ and $\lambda=\frac{1}{2}-\mu$ are eigenvalues of the Schur complement operator $\mathcal{S}$ of the Stokes problem on $\Omega$ with the complex conjugate eigenfunctions $p_{R}$ and $p_{I}$ above. (If $p_{R}=0$ or $p_{I}=0$ then they are not eigenfunctions.) The first part of Theorem 2 states that $\frac{1}{2}$ is an eigenvalue of the Schur complement operator with infinite multiplicity, the eigenfunctions are $p_{R}=2 \operatorname{Re}\left(z^{n}\right)$ and $p_{I}=2 \operatorname{Im}\left(z^{n}\right)$ for $n \geq M$.

3. The eigenvalue problem of $\mathcal{M}_{S} \circ \mathcal{C}$. In this section the conformal mapping $g$ of the unit disc $D$ onto the simply connected domain $\Omega$ is assumed to satisfy the conditions of Theorem 2 . $-\frac{1}{2}$.

LemmA 3. $\Gamma:=\left(a_{1}, 2 a_{2}, \ldots, M a_{M}\right)^{T}$ is an eigenvector of $\mathcal{M}_{S}$ to the eigenvalue

Proof. The matrix $\mathcal{M}_{S}$ can be decomposed as $\mathcal{M}_{S}=-\frac{1}{2} \mathcal{D} \hat{\mathcal{A}} \mathcal{A}^{-1}$ where

$$
\hat{\mathcal{A}}=\left(\begin{array}{cccccc}
a_{1} & a_{2} & a_{3} & \ldots & \ldots & a_{M} \\
a_{2} & a_{3} & \ldots & \ldots & a_{M} & 0 \\
a_{3} & \ldots & \ldots & a_{M} & 0 & 0 \\
\vdots & & & \vdots & \vdots & \vdots \\
a_{M} & 0 & 0 & \ldots & \ldots & 0
\end{array}\right), \mathcal{A}=\left(\begin{array}{ccccc}
a_{1} & 0 & 0 & \ldots & 0 \\
2 a_{2} & a_{1} & 0 & \ldots & 0 \\
3 a_{3} & 2 a_{2} & a_{1} & \ldots & 0 \\
\vdots & & \ddots & \ddots & \vdots \\
M a_{M} & \ldots & \ldots & 2 a_{2} & a_{1}
\end{array}\right)
$$

and $\mathcal{D}=\operatorname{diag}(1,2, \ldots, M)$. So $\mathcal{M}_{S} \mathcal{C} \Gamma=\mu \Gamma$ is equivalent to

$$
\mathcal{D} \hat{\mathcal{A}} \mathcal{C}\left(\mathcal{A}^{-1} \Gamma\right)=(-2 \mu) \mathcal{A}\left(\mathcal{A}^{-1} \Gamma\right)
$$

$\mathcal{D} \hat{\mathcal{A}}$ and $\mathcal{A}$ have the same first column $\Gamma=\left(a_{1}, 2 a_{2}, \ldots, M a_{M}\right)^{T}$. Therefore $\mathcal{A}^{-1} \Gamma=$ $\vec{e}_{1}=(1,0, \ldots, 0)^{T}$ is an eigenvector with eigenvalue $-2 \mu=1$.

REMARK 4. Observe that the eigenvector $\Gamma=\left(a_{1}, 2 a_{2}, \ldots, M a_{M}\right)^{T}$ corresponds to $g^{\prime}(z)$, see $(7)$.

THEOREM 5. For all eigenvalues of the matrix $\mathcal{M}_{S}$ we have $|\mu| \leq \frac{1}{2}$. The eigenvalue $-\frac{1}{2}$ is simple; all other eigenvalues satisfy $0<|\mu|<\frac{1}{2}$. So $\bar{M}_{S}$ is nonsingular.

Proof. The eigenproblem (15) for $p=\left(p_{0}, p_{1}, \ldots, p_{M-1}\right)^{T}$ can be reformulated as follows. Instead of the vector $p$ we introduce the polynomial $p(z)=p_{0}+p_{1} z+\cdots+$ $p_{M-1} z^{M-1}$. We use

$$
\int_{|z|<1} z^{n} \bar{z}^{m} d x d y=\left\{\begin{array}{ccc}
\frac{\pi}{n+1}, & \text { if } & n=m \\
0, & \text { if } & n \neq m
\end{array}\right.
$$


for $n, m=0,1, \ldots$ (see $[15])$. There follows

$$
s_{k}=\frac{1}{\pi} \int_{|z|<1} \bar{z}^{k} \frac{g^{\prime}(z)}{\overline{g^{\prime}(z)}} d x d y
$$

for the quantities $s_{k}(k=0,1, \ldots M-1)$ introduced in (14). We also have for the coefficients of the polynomial $p(z)$ the integral representation

$$
p_{k}=\frac{1}{\pi} \int_{|z|<1}(k+1) \bar{z}^{k} p(z) d x d y .
$$

With this notation, $\left[\mathcal{M}_{S} \mathcal{C} p\right]_{k}=\mu p_{k}$ is equivalent to

$$
\int_{|z|<1}(k+1) \bar{z}^{k} \frac{g^{\prime}(z)}{\overline{g^{\prime}(z)}} \overline{p(z)} d x d y=-2 \mu \int_{|z|<1}(k+1) \bar{z}^{k} p(z) d z .
$$

Multiplying this equation by $\frac{\bar{q}_{k}}{k+1} \in \mathbb{C}$ and summing up over $k=0,1, \ldots M-1$, we get

$$
\int_{|z|<1} \overline{q(z)} \frac{g^{\prime}(z)}{\overline{g^{\prime}(z)}} \overline{p(z)} d x d y=-2 \mu \int_{|z|<1} \overline{q(z)} p(z) d z
$$

where $q(z)=q_{0}+q_{1} z+\cdots+q_{M-1} z^{M-1}$.

So the equivalent formulation of the eigenvalue problem (15) is: find the complex polynomial $p(z)$ of order $M-1$ and the corresponding complex number $\mu$ so that for all complex polynomials $q(z)$ of order $M-1$ there holds

$$
\int_{D} \overline{p(z) q(z)} \frac{g^{\prime}(z)}{g^{\prime}(z)} d x d y=-2 \mu \int_{D} p(z) \overline{q(z)} d x d y .
$$

Substituting now $q(z)=p(z)$ into (22) we have

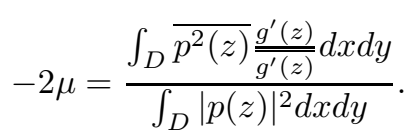

Estimating the integral by taking the absolute value, there follows

$$
2|\mu| \leq \frac{\int_{D}|p(z)|^{2}\left|\frac{g^{\prime}(z)}{\bar{g}^{\prime}(z)}\right| d x d y}{\int_{D}|p(z)|^{2} d x d y}=\frac{\int_{D}|p(z)|^{2} d x d y}{\int_{D}|p(z)|^{2} d x d y}=1 .
$$

Another formulation of (22) can be achieved using the inverse of the (bijective) conformal map $w=g(z)$ and putting $d u d v=\left|g^{\prime}(z)\right|^{2} d x d y$ for the area elements:

$$
\int_{D} \frac{\overline{p(z)}}{\overline{g^{\prime}(z)}} \frac{\overline{q(z)}}{\overline{g^{\prime}(z)}}\left|g^{\prime}(z)\right|^{2} d x d y=-2 \mu \int_{D} \frac{p(z)}{g^{\prime}(z)} \frac{\overline{q(z)}}{\overline{g^{\prime}(z)}}\left|g^{\prime}(z)\right|^{2} d x d y .
$$

Now set

$$
\begin{aligned}
& P(w)=\frac{p\left(g^{-1}(w)\right)}{g^{\prime}\left(g^{-1}(w)\right)}, \\
& Q(w)=\frac{q\left(g^{-1}(w)\right)}{g^{\prime}\left(g^{-1}(w)\right)} .
\end{aligned}
$$


We have then, instead of (22),

$$
\int_{\Omega} \overline{P(w) Q(w)} d u d v=-2 \mu \int_{\Omega} P(w) \overline{Q(w)} d u d v .
$$

Substituting $p(z)=g^{\prime}(z)$ we find that $P(w)=1$ is an eigenfunction to the eigenvalue $\mu=-\frac{1}{2}$. The other eigenfunctions $P(w)$ are orthogonal to this one:

$$
\int_{\Omega} P(w) d u d v=0
$$

So we have the eigenvalue problem of finding $P(w)$ of the form (23) and $\mu$ such that for all $Q(w)$ of the form (24) there holds $(25)$ and (26).

Substituting $Q(w)=P(w)$ into (25) and taking the absolute value, there follows

$$
\left|\int_{\Omega} P(w)^{2} d u d v\right|=2|\mu| \int_{\Omega}|P(w)|^{2} d u d v \text { and } \int_{\Omega} P(w) d u d v=0 .
$$

Now Theorem 2 in Section 6 of [9] - that inequality due to Friedrichs the connection of which to the inf-sup stability of the Stokes problem has been considered in [10], [18], [19] - gives $|\mu|<\frac{1}{2}$. This means that $-\frac{1}{2}$ is a simple eigenvalue of the matrix $\mathcal{M}_{S}$.

REMARK 6. Let us clarify the meaning of the orthogonality (26). Using (20) and the diagonal matrix $\mathcal{D}=\operatorname{diag}(1,2, \ldots, M)$ again, we have

$$
\left(\mathcal{D}^{-1} p, q\right)=\frac{1}{\pi} \int_{|z|<1} p(z) \overline{q(z)} d x d y
$$

for the Euclidean scalar product $(p, q)=\sum_{k=0}^{M-1} p_{k} \bar{q}_{k}$ of the vectors $p=$ $\left(p_{0}, \ldots, p_{M-1}\right)^{T}$ and $q=\left(q_{0}, \ldots, q_{M-1}\right)^{T}$. Because of

$$
\int_{\Omega} P(w) d u d v=\int_{|z|<1} p(z) \overline{g^{\prime}(z)} d x d y
$$

the orthogonality (26) is equivalent to $\left(\mathcal{D}^{-1} p, \Gamma\right)=0$ where $\Gamma$ is the vector already used in Lemma 3.

REMARK 7. From (22) follows that $\mu=-\frac{1}{2}$ is an eigenvalue of (15) with the eigenfunction $p(z)=g^{\prime}(z)$. We have $f(z)=1$ in Theorem 2. So $p_{R}=1$ is an eigenfunction of the Schur complement operator to the eigenvalue 1. But then $p_{I}=0$ and therefore this is not an eigenfunction, hence 1 is a simple eigenvalue.

REMARK 8. The eigenvalue problem (25), (26) is connected with the eigenvalue problem considered in Section 8 in [9]. The only difference is that we now have $\int_{\Omega} \overline{P(w) Q(w)} d u d v$ instead of $\operatorname{Re} \int_{\Omega} P(w) Q(w) d u d v$. Therefore complex eigenvalues are also allowed (but we have a finite number of eigenvalues because of $p(z)$ and $q(z)$ are polynomials in (22)). The underlying operator of this eigenvalue problem is $I-2 \mathcal{M}_{S} \circ \mathcal{C}$ acting on a finite dimensional subspace of the complex Hilbert space $\mathcal{F}$ on $\Omega$ considered in Section 7 of [9].

Now consider the case of complex eigenvalues of (15): $\mu=\mu_{R}+i \mu_{I} \in \mathbb{C}$. We have

$$
\mu f(z)=\left(\mu_{R} \operatorname{Re} f(z)-\mu_{I} \operatorname{Im} f(z)\right)+i\left(\mu_{R} \operatorname{Im} f(z)+\mu_{I} \operatorname{Re} f(z)\right) .
$$


Using again (16) and (17) and the notations $p_{R}=2 \operatorname{Re} f$ and $p_{I}=2 \operatorname{Im} f$, we obtain

$$
\begin{aligned}
\mathcal{S} p_{R} & =\frac{1}{2} p_{R}+\mu_{R} p_{R}-\mu_{I} p_{I}, \\
\mathcal{S} p_{I} & =\frac{1}{2} p_{I}-\mu_{R} p_{I}-\mu_{I} p_{R} .
\end{aligned}
$$

The equations, in block matrix form, are

$$
\left(\begin{array}{cc}
\mathcal{S} & 0 \\
0 & \mathcal{S}
\end{array}\right)\left(\begin{array}{c}
p_{R} \\
p_{I}
\end{array}\right)=\left(\begin{array}{cc}
\frac{1}{2}+\mu_{R} & -\mu_{I} \\
-\mu_{I} & \frac{1}{2}-\mu_{R}
\end{array}\right)\left(\begin{array}{l}
p_{R} \\
p_{I}
\end{array}\right) .
$$

We have two real eigenvalues of the right-hand side matrix of (27):

$$
\tilde{\mu}_{1,2}=\frac{1}{2} \pm \sqrt{\mu_{R}^{2}+\mu_{I}^{2}}=\frac{1}{2} \pm|\mu| .
$$

Because $\mathcal{M}_{S}$ is a non-singular matrix, we have $\mu \neq 0$, and we can transform the right-hand side matrix in (27) to diagonal form using

$$
T:=\frac{1}{2}\left(\begin{array}{cc}
-\frac{\mu_{I}}{|\mu|} & 1-\frac{\mu_{R}}{|\mu|} \\
\frac{\mu_{I}}{|\mu|} & 1+\frac{\mu_{R}}{|\mu|}
\end{array}\right) .
$$

So (27) can be reformulated:

$$
\left(\begin{array}{cc}
\mathcal{S} & 0 \\
0 & \mathcal{S}
\end{array}\right)\left(T\left(\begin{array}{c}
p_{R} \\
p_{I}
\end{array}\right)\right)=\left(\begin{array}{cc}
\frac{1}{2}+|\mu| & 0 \\
0 & \frac{1}{2}-|\mu|
\end{array}\right)\left(T\left(\begin{array}{c}
p_{R} \\
p_{I}
\end{array}\right)\right)
$$

Therefore, the following equations hold:

$$
\begin{aligned}
& \mathcal{S} \tilde{p}_{R}=\left(\frac{1}{2}+|\mu|\right) \tilde{p}_{R} \\
& \mathcal{S} \tilde{p}_{I}=\left(\frac{1}{2}-|\mu|\right) \tilde{p}_{I}
\end{aligned}
$$

where $\left(\begin{array}{c}\tilde{p}_{R} \\ \tilde{p}_{I}\end{array}\right)=T\left(\begin{array}{c}p_{R} \\ p_{I}\end{array}\right)$. These eigenfunctions are

$$
\begin{aligned}
& \tilde{p}_{R}=-\frac{\mu_{I}}{|\mu|} \operatorname{Re} \frac{p(z)}{g^{\prime}(z)}+\left(1-\frac{\mu_{R}}{|\mu|}\right) \operatorname{Im} \frac{p(z)}{g^{\prime}(z)}, \\
& \tilde{p}_{I}=\frac{\mu_{I}}{|\mu|} \operatorname{Re} \frac{p(z)}{g^{\prime}(z)}+\left(1+\frac{\mu_{R}}{|\mu|}\right) \operatorname{Im} \frac{p(z)}{g^{\prime}(z)} .
\end{aligned}
$$

Let us summarize the results of this section:

Corollary 9. Every eigenvalue $\mu \neq-\frac{1}{2}$ of the matrix $\mathcal{M}_{S}$ gives rise to two additional eigenvalues of the Schur complement operator $\mathcal{S}$. These eigenvalues are

- $\frac{1}{2} \pm \mu \in(0,1)$, if $\mu$ is real (with the eigenfunctions (18), (19));

- $\frac{1}{2} \pm|\mu| \in(0,1)$ if $\mu$ is not real (with the eigenfunctions (30), (31)).

(The case of the eigenvalue $\mu=-\frac{1}{2}$ has already been considered in Remark \%.)

Let us clarify the meaning of "additional eigenvalues of $\mathcal{S}$ " in Corollary 9. In case $\Omega$ is a disc, the only eigenvalue of $\mathcal{S}$ in $(0,1)$ is $\frac{1}{2}$ which is of infinite multiplicity. By 
the first part of Theorem 2 the eigenvalue $\lambda=\frac{1}{2}$ remains an eigenvalue of infinite multiplicity in case $\Omega$ is a polynomial conformal map of $D$. But for such domains $\Omega$ the Schur complement operator $\mathcal{S}$ has further a finite number of eigenvalues in $(0,1)$ as explained in Corollary 9. These additional eigenvalues - and eigenfunctions - can be computed with the help of the matrix $\mathcal{M}_{S}$.

REMARK 10. An important consequence of Corollary 9 is that the Crouzeix-Velte subspace investigated in [16] is not reduced to zero for domains arising as conformal maps of the unit disc by a polynomial mapping function.

REMARK 11. If $g(z)$ has real coefficients then the domain $\Omega$ is symmetrical to the real axis and $\mathcal{M}_{S} \in \mathbb{R}^{M \times M}$. In this case (15) is equivalent to

$$
\mathcal{D}^{-\frac{1}{2}} \mathcal{M}_{S} \mathcal{D}^{\frac{1}{2}} w=-2 \mu w
$$

where $w=\mathcal{D}^{-\frac{1}{2}} p$ with the real symmetric matrix $\mathcal{D}^{-\frac{1}{2}} \mathcal{M}_{S} \mathcal{D}^{\frac{1}{2}}$. So all eigenvalues of $\mathcal{M}_{S}$ are real. (If we calculate these eigenvalues numerically it is worth to transform $\mathcal{M}_{S}$ to this form.)

Remark 12. Denote $\mu_{2}:=\max \left\{|\mu|: \mu \in \sigma\left(\mathcal{M}_{S}\right) \backslash\left\{-\frac{1}{2}\right\}\right\}$. As a consequence of Corollary 9 we have $\mu_{2}<\frac{1}{2}$ and we can compute the inf-sup stability constant (3) of the domain $\Omega$ :

$$
\beta_{0}^{2}(\Omega)=\frac{1}{2}-\mu_{2}
$$

Now let us give some examples with special mapping functions $g(z)$, compute the entries (14) of the matrix (13) and the eigenvalues.

1. In case $M=2$ and $g(z)=a_{1} z+a_{2} z^{2}, a_{1} \neq 0$ we have $b_{0}=\frac{1}{a_{1}}$ and $b_{1}=-\frac{2 a_{2}}{a_{1}^{2}}$ and

$$
\mathcal{M}_{S}=-\frac{1}{2}\left(\begin{array}{cc}
\frac{\left|a_{1}\right|^{2}-2\left|a_{2}\right|^{2}}{\bar{a}_{2}^{2}} & \frac{a_{2}}{\bar{a}_{1}} \\
2 \frac{a_{2}}{\bar{a}_{1}} & 0
\end{array}\right) .
$$

The eigenvalues of (15) are $\mu_{1}=-\frac{1}{2}$ with the eigenvector $\left(a_{1}, 2 a_{2}\right)^{T}$, and $\mu_{2}=\left(\frac{a_{2}^{2}}{\bar{a}_{1}^{2}}\right)$ with the eigenvector $\left(\bar{a}_{2},-\bar{a}_{1}\right)^{T}$.

Using $g^{\prime}\left(-\frac{a_{1}}{2 a_{2}}\right)=0$ and $g^{\prime}(z) \neq 0$ in $|z| \leq 1$ there follows $\left|\frac{a_{1}}{2 a_{2}}\right|>1$. This gives $\left|\mu_{2}\right|<\frac{1}{4}$. Moreover for $a_{1}=1$ and $\left|a_{2}\right| \rightarrow \frac{1}{2}$ we have $\mu_{2} \rightarrow \frac{1}{4}$. This means that for $M=2$ the assumption $g^{\prime}(z) \neq 0$ on $\bar{D}$ of Theorem 2 can be weakened. $g^{\prime}(z) \neq 0$ in $D$, but $g^{\prime}\left(z_{0}\right)=0$ with $g^{\prime \prime}\left(z_{0}\right) \neq 0$ can happen for a $z_{0} \in \partial D$. (In this case we have an inner angle of $2 \pi-$ an internal cusp at the point $g\left(z_{0}\right) \in \partial \Omega$.) We have now $\beta_{0}^{2}(\Omega)=\frac{1}{2}-\left|\frac{a_{2}}{a_{1}}\right|^{2}$ for the inf-sup stability constant from Remark 12 .

2. In case $g(z)=z-\frac{c}{M} z^{M}(c \in \mathbb{R}, M>1$ and integer, as earlier), which is univalent in $D$ for $|c| \leq 1$, we have

$$
\mathcal{M}_{S}=-\frac{1}{2}\left(\begin{array}{ccccc}
1-\frac{c^{2}}{M} & 0 & \ldots & 0 & -\frac{c}{M} \\
0 & 0 & \ldots & -\frac{2 c}{M} & 0 \\
\vdots & \vdots & \vdots & \vdots & \vdots \\
0 & -\frac{(M-1) c}{M} & \ldots & 0 & 0 \\
-\frac{M c}{M} & 0 & \ldots & 0 & 0
\end{array}\right)
$$


The eigenvalues are $-\frac{1}{2},-\frac{c}{2 M} \sqrt{k(M-k+1)}$ for $k=2, \ldots, M-1$ and $\frac{c^{2}}{2 M}$. We have here the inf-sup constant value from Remark 12:

$$
\beta_{0}^{2}(\Omega)= \begin{cases}\frac{1}{2}-\frac{|c|}{4}\left(1+\frac{1}{M}\right) & \text { for } M \text { odd } \\ \frac{1}{2}-\frac{|c|}{4} \sqrt{1+\frac{2}{M}} & \text { for } M \text { even. }\end{cases}
$$

Example 1 implies that the assumptions on the first derivative of the mapping polynomial $g$ in Theorem 2 can be weakened. We first cite the following result from [11]:

Lemma 13. (Lemma 6 in [11]) Let $g(z)$ be holomorphic and univalent in $D$ and holomorphic in $z_{0} \in \partial D$, and $g^{\prime}\left(z_{0}\right)=0$. Then $g^{\prime \prime}\left(z_{0}\right) \neq 0$ follows.

Using this result we can formulate the following

LEMma 14. Let $g$ be a polynomial conformal mapping $D \rightarrow \Omega$ of the form (7), let $g$ be univalent in $\bar{D} ; g^{\prime}(z) \neq 0$ in $D$. Then $|\mu| \leq \frac{1}{2}$ holds for the eigenvalues of (15). Theorem 2 and Corollary 9 remain valid.

Proof. Because the polynomial $g$ is holomorphic on $\bar{D}$, Lemma 13 holds, and so we have $g^{\prime \prime}\left(z_{0}\right) \neq 0$ if $g^{\prime}\left(z_{0}\right)=0$ in $z_{0} \in \partial D$. The proof is similar to that of Theorem 2. Set $p(z):=\sum_{n=0}^{M-1} p_{n} z^{n}$ and $u_{0}(z):=\frac{1}{2} g(z) \frac{\overline{p(z)}}{g^{\prime}(z)}$ which is by Lemma 1 a solution of the momentum equation (1). $u_{0}$ has boundary values in the points $z \in \partial D$ where $g^{\prime}(z) \neq 0$. If $g^{\prime}\left(z_{0}\right)=0$ but $g^{\prime \prime}\left(z_{0}\right) \neq 0$ for $z_{0} \in \partial D$ then we have in a neighbourhood of $z_{0}$

$$
g(z)=\tilde{a}_{0}+\tilde{a}_{2}\left(z-z_{0}\right)^{2}+\cdots+\tilde{a}_{M}\left(z-z_{0}\right)^{M},
$$

with $\tilde{a}_{0}=g\left(z_{0}\right)$ and $\tilde{a}_{2}=\frac{1}{2} g^{\prime \prime}\left(z_{0}\right) \neq 0$. We further have

$$
\begin{aligned}
\frac{g(z)}{\overline{g^{\prime}(z)}=} & \frac{\tilde{a}_{0}}{\left(\bar{z}-\bar{z}_{0}\right) \overline{2 \tilde{a}_{2}+3 \tilde{a}_{3}\left(z-z_{0}\right)+\cdots+M \tilde{a}_{M}\left(z-z_{0}\right)^{M-2}}}+ \\
& \left(z-z_{0}\right) \cdot e^{2 i \arg \left(z-z_{0}\right)} \frac{\tilde{a}_{2}+\tilde{a}_{3}\left(z-z_{0}\right)+\cdots+\tilde{a}_{M}\left(z-z_{0}\right)^{M-2}}{\overline{2 \tilde{a}_{2}+3 \tilde{a}_{3}\left(z-z_{0}\right)+\cdots+M \tilde{a}_{M}\left(z-z_{0}\right)^{M-2}}}
\end{aligned}
$$

Thus in case $\tilde{a}_{0}=g\left(z_{0}\right)=0$ there follows $u\left(z_{0}\right)=\lim _{z \rightarrow z_{0}} u(z)=0$, but $u_{0}$ does not have a boundary value in $z_{0}$ for

$$
g\left(z_{0}\right) \neq 0, g^{\prime}\left(z_{0}\right)=0 \text { and } g^{\prime \prime}\left(z_{0}\right) \neq 0 .
$$

We add a holomorphic and a conjugate holomorphic function so that $u_{0}(z)+v_{1}(z)+$ $\overline{v_{2}(z)}$ fulfills the homogenous boundary condition - except for points with the properties (33):

$$
\begin{aligned}
& v_{1}(z):=-\frac{1}{2} \sum_{k=1}^{M}\left[\sum_{m-n=k} a_{m}\left(\sum_{\ell=0}^{n} \overline{b_{n-\ell} p_{\ell}}\right)\right] z^{k} ; \\
& \overline{v_{2}(z)}:=-\frac{1}{2} \sum_{k=0}^{\infty}\left[\sum_{n-m=k} a_{m}\left(\sum_{\ell=0}^{n} \overline{b_{n-\ell} p_{\ell}}\right)\right] \bar{z}^{k} .
\end{aligned}
$$

$v_{1}(z)$ is a polynomial, and $\overline{v_{2}(z)}=-u_{0}(z)-v_{1}(z)$ on $\partial D$. Hence the series form of $v_{2}(z)$ converges in $D$ and has an extension onto $\partial D$ which is continuous on $\partial D$ 
except in a finite number of points. We have (11) and (12) for the coefficients in the expansion $v_{1}^{\prime}=\sum_{k=0}^{M-1} q_{k} z^{k}$.

Now we follow the proof of Theorem 5 above. For $0<r<1$,

$$
\frac{1}{\pi} \int_{|z|<r} \bar{z}^{k} \frac{g^{\prime}(z)}{\overline{g^{\prime}(z)}} d x d y=\sum_{n=0}^{M-(k+1)} a_{n+k+1} \bar{b}_{n} r^{2(n+k)}
$$

because of the assumptions on $g$. Taking the limit for $r \rightarrow 1$ from below, there follows for $k=0,1, \ldots M-1$

$$
\lim _{r \rightarrow 1-} \sum_{n=0}^{M-(k+1)} a_{n+k+1} \bar{b}_{n} r^{2(n+k)}=s_{k},
$$

and then

$$
s_{k}=\lim _{r \rightarrow 1-} \frac{1}{\pi} \int_{|z|<r} \bar{z}^{k} \frac{g^{\prime}(z)}{\overline{g^{\prime}(z)}} d x d y
$$

Because of $\sup _{|z|<r}\left|\bar{z}^{k} \frac{g^{\prime}(z)}{g^{\prime}(z)}\right|<r^{k}<1$, we finally obtain (21). From here we get the eigenvalue problem (22) and the proof ends as that of Theorem 5. Therefore Corollary 9 remains valid in this case, too. $\square$

REMARK 15 . The boundary $\partial \Omega$ has an inner angle of $2 \pi$ (an internal cusp) in $g\left(z_{0}\right)$, for points $z_{0} \in \partial D$ involved in Lemma 13 and Lemma 14 .

4. The case of non-polynomial mappings. Now we turn to a generalization of Theorem 2 to power series instead of polynomials:

TheOREm 16. Let $\Omega$ be a simply connected plane domain. Let the bijective conformal map $D \rightarrow \Omega$ be of the form

$$
g(z)=\sum_{m=0}^{\infty} a_{m} z^{m}
$$

where this series converges on an open neighbourhood of $\bar{D}$. Suppose further $g^{\prime}(z) \neq 0$ in $\bar{D} ; \frac{1}{g^{\prime}(z)}=\sum_{\ell=0}^{\infty} b_{\ell} z^{\ell}$. Let be $f(z)=\frac{\sum_{n=0}^{\infty} p_{n} z^{n}}{g^{\prime}(z)}$. Then we have for the Schur complement operator $\mathcal{S}$ of the Stokes-equation:

- $p_{R}=2$ Ref leads to the divergence $\mathcal{S} p_{R}=\frac{1}{2} p_{R}+2 \operatorname{Re} \frac{\sum_{n=0}^{\infty} q_{n} z^{n}}{g^{\prime}(z)}$,

- $p_{I}=2 \operatorname{Im} f$ leads to the divergence $\mathcal{S} p_{I}=\frac{1}{2} p_{I}-2 \operatorname{Im} \frac{\sum_{n=0}^{\infty} q_{n} z^{n}}{g^{\prime}(z)}$.

The coefficients $s_{k, \ell}$ of the conjugate linear operator $\left(p_{\ell}\right)_{\ell=0,1, \ldots} \mapsto\left(q_{k}\right)_{k=0,1, \ldots}$ are defined by

$$
s_{k, \ell}=-\frac{k+1}{2} \sum_{m=\ell+k+1}^{\infty} a_{m} \bar{b}_{m-(\ell+k+1)}
$$

for $k, \ell=0,1, \ldots$ 
Proof. The proof is very similar to that of Theorem 2. First we construct a special solution of the homogeneous momentum equation (1) as described by Lemma 1:

$$
\begin{aligned}
u_{0}(z)= & \frac{1}{2} g(z) \overline{f(z)}=\frac{1}{2} \sum_{k=0}^{\infty}\left\{\sum_{m=0}^{\infty} a_{m}\left(\sum_{\ell=0}^{m+k} \bar{b}_{m+k-\ell} \bar{p}_{\ell}\right) z^{m} \bar{z}^{m+k}\right\}+ \\
& \frac{1}{2} \sum_{k=1}^{\infty}\left\{\sum_{m=k}^{\infty} a_{m}\left(\sum_{\ell=0}^{m-k} \bar{b}_{m-k-\ell} \bar{p}_{\ell}\right) z^{m} \bar{z}^{m-k}\right\}
\end{aligned}
$$

We add a holomorphic and a conjugate holomorphic function to fulfill the homogenous boundary condition. The boundary $\partial D$ of the unit disc is given by $\left.z \bar{z}\right|_{\partial D}=1$. The conjugate holomorphic function

$$
\overline{v_{2}(z)}=-\frac{1}{2} \sum_{k=0}^{\infty}\left\{\sum_{m=0}^{\infty} a_{m}\left(\sum_{\ell=0}^{m+k} \bar{b}_{m+k-\ell} \bar{p}_{\ell}\right)\right\} \bar{z}^{k}
$$

has divergence 0 . The derivative of the holomorphic function

$$
v_{1}(z)=-\frac{1}{2} \sum_{k=1}^{\infty}\left\{\sum_{m=k}^{\infty} a_{m}\left(\sum_{\ell=0}^{m+k} \bar{b}_{m+k-\ell} \bar{p}_{\ell}\right)\right\} z^{k}
$$

has the expansion $v_{1}^{\prime}(z)=\sum_{k=0}^{\infty} q_{k} z^{k}$ where

$$
q_{k}=-\frac{k+1}{2} \sum_{\ell=0}^{\infty}\left(\sum_{m=\ell+k+1}^{\infty} a_{m} \bar{b}_{m-(\ell+k+1)}\right) \bar{p}_{\ell} .
$$

Now the proof is completed as that of Theorem 2 by using Lemma 1 .

The convergence of the power series in this proof is a consequence of a result in [15]: if the power series $A(z)=\sum_{n=0}^{\infty} a_{n} z^{n}$ and $B(z)=\sum_{n=0}^{\infty} b_{n} z^{n}$ both converge for $|z|<R$, then the series $C(z)=\sum_{n=0}^{\infty} c_{n} z^{n}$, where $c_{n}=\sum_{\ell=0}^{n} a_{\ell} b_{n-\ell}$, also converges for $|z|<R$ and $C(z)=A(z) B(z)$.

REMARK 17. The convergence property of the series (34) in Theorem 16 is not fulfilled for every mapping function (see for example the mapping onto a half plane). However the statement of the theorem remains valid for mappings with convergent series form in $D$ and with the additional property:

$$
\frac{1}{g^{\prime}(z)}=\sum_{\ell=0}^{N} b_{\ell} z^{\ell}
$$

This means that the reciprocial of the derivative is in fact a polynomial. In this case we have instead of (35) the formula

$$
s_{k, \ell}=-\frac{k+1}{2} \sum_{m=\ell+k+1}^{N+\ell+k+1} a_{m} \bar{b}_{m-(\ell+k+1)} .
$$

REMARK 18. Let us define the space $\ell_{(2,-1 / 2)}$ of all complex sequences $\left(p_{k}\right)_{k=0}^{\infty}$ for which $\sum_{k=0}^{\infty} \frac{\left|p_{k}\right|^{2}}{k+1}<\infty$. The inner product of two sequences from this space is 
defined by

$$
(p, q)_{2}:=\sum_{k=0}^{\infty} \frac{p_{k} \bar{q}_{k}}{k+1}
$$

and the norm of a sequence is $\|p\|_{2}=\sqrt{(p, p)_{2}}$. This $\ell_{(2,-1 / 2)}$ space is a Hilbert space (see e.g. [15]). Moreover, using (20), there follows

$$
(p, q)_{2}=\frac{1}{\pi}(p, q)_{0},
$$

where $p(z)=\sum_{k=0}^{\infty} p_{k} z^{k} \in L_{2}(D)$ and $q(z)=\sum_{k=0}^{\infty} q_{k} z^{k} \in L_{2}(D)$ and $(p, q)_{0}=$ $\int_{D} p(z) \overline{q(z)} d x d y$ is the usual inner product of the space $L_{2}(D)$. In the following we also use the notation $\|p\|_{0}=\sqrt{(p, p)_{0}}$ for the $L_{2}$ norm of a function $p$.

The mapping $\left(p_{\ell}\right)_{\ell=0,1, \ldots} \mapsto\left(q_{k}\right)_{k=0,1, \ldots}$ in Theorem 16 between the coeffitients of the Taylor series of $p(z)$ and the derivative $v_{1}^{\prime}(z)$ of (36) is conjugate linear.

In order to prove that this mapping is also bounded, let us define the transformation

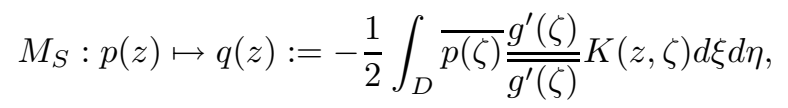

for $p(z)=\sum_{k=0}^{\infty} p_{k} z^{k} \in L_{2}(D)$, where we have used the notation $\zeta=\xi+i \eta$, and $K(z, \zeta)=\frac{1}{\pi} \frac{1}{(1-\zeta z)^{2}}$ is the Bergman kernel function of the unit disc (see [15]). Because $p, K \in L_{2}(D)$ and the modulus of $\frac{g^{\prime}(\zeta)}{g^{\prime}(\zeta)}$ is 1 , there follows $q \in L_{2}(D)$. Let us compute the $L_{2}$ norm of $q$. We multiply (39) by $\overline{q(z)}$ and integrate over $D$ :

$$
\int_{D}|q(z)|^{2} d x d y=-\frac{1}{2} \int_{D} \overline{q(z)} \int_{D} \overline{p(\zeta)} \frac{g^{\prime}(\zeta)}{\overline{g^{\prime}(\zeta)}} K(z, \zeta) d \xi d \eta d x d y
$$

Changing the order of the integration gives

$$
\int_{D}|q(z)|^{2} d x d y=-\frac{1}{2} \int_{D} \overline{p(\zeta)} \frac{g^{\prime}(\zeta)}{\overline{g^{\prime}(\zeta)}}\left(\int_{D} K(z, \zeta) \overline{q(z)} d x d y\right) d \xi d \eta .
$$

Using the reproducing property $\overline{q(\zeta)}=\int_{D} K(z, \zeta) \overline{q(z)} d x d y$ of the Bergman kernel there follows

$$
\int_{D}|q(z)|^{2} d x d y=-\frac{1}{2} \int_{D} \overline{p(\zeta) q(\zeta)} \frac{g^{\prime}(\zeta)}{\overline{g^{\prime}(\zeta)}} d \xi d \eta .
$$

We estimate this by taking the absolute value and using the Cauchy-Schwarz inequality:

$$
\|q\|_{0}^{2} \leq \frac{1}{2} \int_{D}|p(z)| \cdot|q(z)| d x d y \leq \frac{1}{2}\|p\|_{0}\left\|_{q}\right\|_{0}
$$

Therefore we have $\|q\|_{0} \leq \frac{1}{2}\|p\|_{0}$, i.e. the continuity of the integral transformation $M_{S}$.

Let the function $q$ have the series expansion $q(z)=\sum_{k=0}^{\infty} q_{k} z^{k}$. Investigating the series forms of $p$ and $q$, which are convergent in $D$ we find

$$
q_{k}=\left[\mathcal{M}_{S} \mathcal{C} p\right]_{k}
$$


where $p$ also denotes the infinite vector composed of the coefficients of the Taylor series expansion of $p(z), \mathcal{C}$ is the usual conjugacy and $\mathcal{M}_{S}$ is an infinite dimensional matrix acting on the space $\ell_{(2,-1 / 2)}$. The elements of this matrix are $s_{k, \ell}=-\frac{k+1}{2} s_{k+\ell}$ for $k, \ell=0,1, \ldots$, where

$$
s_{k}:=\sum_{m=0}^{\infty} a_{m+k+1} \bar{b}_{m}
$$

Using that the integral transformation $M_{S}$ is bounded and (38), we see that the conjugate linear operator $\mathcal{M}_{S} \circ \mathcal{C}$ is also bounded. Moreover, by the correspondence between the integral transformation $M_{S}$ and $\mathcal{M}_{S} \circ \mathcal{C}$, there follows for the quantities (40) the integral representation

$$
s_{k}=\frac{1}{\pi} \int_{D} \bar{z}^{k} \frac{g^{\prime}(z)}{\frac{g^{\prime}(z)}{\prime}} d x d y
$$

which is in fact (21). We obtain by estimation

$$
\left|s_{k}\right| \leq \frac{1}{\pi} \int_{0}^{2 \pi} \int_{0}^{1} \rho^{k} \rho d \rho d \theta=\frac{2}{k+2},
$$

and therefore $\left|s_{k, \ell}\right| \leq \frac{k+1}{k+\ell+2}$.

The infinite matrix $\mathcal{M}_{S}$ has the special form

$$
\mathcal{M}_{S}=-\frac{1}{2}\left(\begin{array}{cccccc}
s_{0} & s_{1} & s_{2} & \ldots & s_{k} & \ldots \\
2 s_{1} & 2 s_{2} & \ldots & 2 s_{k} & \ldots & \ldots \\
3 s_{2} & \ldots & 3 s_{k} & \ldots & \ldots & \ldots \\
\vdots & \vdots & \vdots & \vdots & \vdots & \vdots \\
(k+1) s_{k} & \ldots & \ldots & \ldots & \ldots & \ldots \\
\vdots & \vdots & \vdots & \vdots & \vdots & \vdots
\end{array}\right)
$$

similar to the case investigated in Section 3. The additional eigenvalues of the Schur complement operator are as in Corollary 9.

We can give an integral form of the eigenvalue problem $\mathcal{M}_{S} \mathcal{C} p=\mu p$ similar to (22): find $\mu$ and the convergent power series $p(z)=\sum_{m=0}^{\infty} p_{m} z^{m}$ such that (22) is valid for all convergent $q(z)=\sum_{m=0}^{\infty} q_{m} z^{m}$. The integral formulation (25) carries over to this case, too. (But now the eigenvalue problem is not finite dimensional, although again equivalent to the eigenvalue problem considered in [9].) If the infinite vector composed of the coefficients of $g^{\prime}(z)$ is an element of $\ell_{(2,-1 / 2)}$, then it is an eigenvector to the eigenvalue $-\frac{1}{2}$. The absolute value of other eigenvalues is less than $\frac{1}{2}$, and the eigenfunctions are orthogonal to $g^{\prime}(z)$ in the sense of Remark 6. (Observe further that $\left(\mathcal{D}^{-1} p, \Gamma\right)=(p, \Gamma)_{2}$, where $\mathcal{D}=\operatorname{diag}(1,2, \ldots)$.)

We again discuss some examples.

1. We take first $g(z)=\frac{a z+b}{c z+d}$ where $c, d \neq 0$ and $a d-b c \neq 0$. We have

$$
g(z)=\frac{a}{c}-\frac{a d-b c}{c d}+\frac{a d-b c}{c d} \sum_{k=1}^{\infty}(-1)^{k+1} \frac{c^{k}}{d^{k}} z^{k}
$$


This series is convergent for $|z|<\left|\frac{d}{c}\right|$ in which the open unit disc is contained for $|c| \leq|d|$. We also have (see Remark 17)

$$
\frac{1}{g^{\prime}(z)}=\frac{d^{2}}{a d-b c}+\frac{2 c d}{a d-b c} z+\frac{c^{2}}{a d-b c} z^{2} .
$$

There follows $a_{1}=\frac{a d-b c}{c d} \cdot \frac{c}{d}$, and $a_{k}=\left(-\frac{c}{d}\right)^{k-1} a_{1}$ for $k \geq 2$. Further $b_{0}=\frac{d^{2}}{a d-b c}, b_{1}=\frac{2 c d}{a d-b c}, b_{2}=\frac{c^{2}}{a d-b c}$ and $b_{k}=0$ for $k \geq 3$. Computing from here $s_{k}$ figuring in the matrix $\mathcal{M}_{S}$, we get

$$
s_{0}=\frac{(a d-b c)\left(|c|^{2}-|d|^{2}\right)^{2}}{d^{4} \overline{a d-b c}}
$$

and

$$
s_{k}=(-1)^{k}\left(\frac{c}{d}\right)^{k} s_{0} \text { for } k=1,2, \ldots
$$

In case $|c|=|d|$ there follows that $\mathcal{M}_{S}$ is the (infinite) zero matrix; we have no additional eigenvalues of the Schur complement operator $\mathcal{S}$.

In case $|c|<|d|$ we have

$$
\mathcal{M}_{S}=-\frac{s_{0}}{2}\left(\begin{array}{cccccc}
1 & q & q^{2} & \ldots & q^{k-1} & \ldots \\
2 q & 2 q^{2} & 2 q^{3} & \ldots & 2 q^{k} & \ldots \\
\vdots & \vdots & \vdots & \vdots & \vdots & \vdots \\
k q^{k-1} & k q^{k} & k q^{k+1} & \ldots & k q^{2 k-2} & \ldots \\
\vdots & \vdots & \vdots & \vdots & \vdots & \vdots
\end{array}\right)
$$

where $q=-\frac{c}{d}$. Let be $u=\left(u_{0}, u_{1}, \ldots\right)^{T} \in \ell_{(2,-1 / 2)}$. We have

$$
\mathcal{M}_{S} \mathcal{C} u=-\frac{s_{0}}{2}\left(\sum_{k=0}^{\infty} \bar{u}_{k} q^{k}\right)\left(1,2 q, \ldots, k q^{k-1}, \ldots\right)^{T}
$$

and here $\mathcal{M}_{S} \mathcal{C} u \in \ell_{(2,-1 / 2)}$ because of $|q|<1$. We see that the operator $\mathcal{M}_{S}$ is rank one and its range contains only $\left(1,2 q, \ldots, k q^{k-1}, \ldots\right)^{T}$. Therefore $\mathcal{M}_{S}$ has only this eigenvector with the eigenvalue

$$
\mu=-\frac{s_{0}}{2} \frac{1}{\left(1-|q|^{2}\right)^{2}}=-\frac{1}{2} \cdot \frac{a d-b c}{\overline{a d-b c}} \cdot \frac{|d|^{4}}{d^{4}} .
$$

We have $|\mu|=\frac{1}{2}$, and there is no additional eigenvalue of the Schur complement operator. We have therefore $\beta_{0}^{2}=\frac{1}{2}$.

Especially we can choose the Blaschke-function $g(z)=\frac{z-\gamma}{1-\bar{\gamma} z},|\gamma|<1$ mapping the unit disc onto itself. We have here $q=\bar{\gamma},|q|<1$. A similar function $g(z)=\frac{\gamma-z \bar{\gamma}}{1-z}, \operatorname{Im} \gamma>0$ maps the unit disc onto the upper half plane $\operatorname{Im} w>0$. We have here $q=1$ and the series form of $g(z)$ does not converge on the whole unit circle but only inside the unit disc. But $g(z)=\gamma+2 i \operatorname{Im} \gamma \sum_{n=1}^{\infty} z^{n}$ and $\frac{1}{g^{\prime}(z)}=\frac{1-2 z+z^{2}}{2 i \operatorname{Im} \gamma}$, and $s_{k}=0$ follows for all $k=0,1, \ldots$ This means that $\mathcal{M}_{S}$ is the zero infinite matrix and we have only the eigenvalues $0, \frac{1}{2}, 1$ of the Schur complement operator. 
2. The transformation $g(z)=\frac{4 a}{(1+z)^{2}}, a>0$ maps the unit disc $|z|<1$ onto the exterior of the parabola $v^{2}=4 a(a-u)$ (i.e. that region which does not contain the focus of the parabola). Now we have the power series form of the mapping

$$
g(z)=\sum_{k=1}^{\infty} 4 a(-1)^{k-1} k z^{k-1}
$$

and $\frac{1}{g^{\prime}(z)}=-\frac{1}{8 a}\left(1+3 z+3 z^{2}+z^{3}\right)$ which is again a polynomial (see Remark 17). Therefore we have

$$
a_{k}=4 a(-1)^{k}(k+1) \text { for } k \geq 0
$$

and $b_{0}=b_{3}=-\frac{1}{8 a}, b_{1}=b_{2}=-\frac{3}{8 a}, b_{\ell}=0$ for $\ell \geq 4$. A simple calculation shows $s_{k}=0$ for $k \geq 0$, so (41) is an infinite zero matrix; we do not have any additional eigenvalues of the Schur complement operator. This example shows that there are domains different from the circle and the halfplane with the Schur complement eigenvalues $0, \frac{1}{2}, 1$. Further we have $\beta_{0}^{2}=\frac{1}{2}$ for the inf-sup constant.

3. The next example is $g(z)=\int_{0}^{z} \frac{1}{\sqrt{1-t^{4}}} d t$ mapping the unit disc onto a square. We have the power series forms which converge for $|z| \leq 1$ :

$$
\begin{aligned}
& g(z)=\sum_{m=0}^{\infty} \frac{c_{m}}{4 m+1} z^{4 m+1} \\
& \frac{1}{g^{\prime}(z)}=\sqrt{1-z^{4}}=1-\frac{1}{2} \sum_{n=1}^{\infty} \frac{c_{n-1}}{n} z^{4 n}
\end{aligned}
$$

where $c_{0}=1, c_{n}=\frac{2 n-1}{2 n} c_{n-1}$. We can give explicit formulae for $c_{n}(n=$ $0,1, \ldots)$ :

$$
c_{n}=\frac{1 \cdot 3 \cdot \ldots(2 n-1)}{2^{n} n !}=\frac{\Gamma\left(n+\frac{1}{2}\right)}{\sqrt{\pi} n !} .
$$

We compute the elements of the infinite matrix $\mathcal{M}_{S}$ :

$$
\begin{aligned}
s_{4 k} & =\frac{1}{4 k+1} c_{k}-\frac{1}{2} \sum_{n=1}^{\infty} \frac{c_{n+k} c_{n-1}}{n(4(n+k)+1)}, \\
s_{4 k+1} & =s_{4 k+2}=s_{4 k+3}=0 \text { for } k=0,1, \ldots
\end{aligned}
$$

Using Barnes's extended hypergeometric function, the formula

$$
s_{4 k}=a_{4 k+1}-\frac{1}{2} a_{4(k+1)+1} F_{k}
$$

holds, where $a_{4 k+1}=\frac{c_{k}}{4 k+1}$ and

$$
F_{k}=F\left(\left[\frac{5}{4}+k, \frac{3}{2}+k, \frac{1}{2}, 1\right],\left[\frac{9}{4}+k, 2+k, 2\right], 1\right) .
$$


This matrix acts on three orthogonal subspaces of $\ell_{(2,-1 / 2)}$. These subspaces contain vectors of the form

$$
\begin{aligned}
\left(v_{0}, 0,0,0 ; v_{4}, 0,0,0 ; v_{8}, 0,0,0 ; \ldots\right)^{T} & \in \ell_{(2,-1 / 2)} \\
\left(0,0, v_{2}, 0 ; 0,0, v_{6}, 0 ; 0,0, v_{10}, 0 ; \ldots\right)^{T} & \in \ell_{(2,-1 / 2)} \\
\left(0, v_{1}, 0, v_{3} ; 0, v_{5}, 0, v_{7} ; 0, v_{9}, 0, v_{11} ; \ldots\right)^{T} & \in \ell_{(2,-1 / 2)}
\end{aligned}
$$

The $\ell_{(2,-1 / 2)}$-vector corresponding to the derivative $g^{\prime}(z)$ of the mapping function is contained in the first subspace:

$$
\left(c_{0}, 0,0,0 ; c_{1}, 0,0,0 ; c_{2}, 0,0,0 ; \ldots\right)^{T} .
$$

The second subspace contains $\ell_{(2,-1 / 2)}$-vectors which correspond to eigenfunctions of the form $v(z)=z^{2}\left(v_{2}+v_{6} z^{4}+\ldots\right)$.

If an eigenvector $\left(0, v_{1}, 0, v_{3} ; 0, v_{5}, 0, v_{7} ; 0, v_{9}, 0, v_{11} ; \ldots\right)^{T} \in \ell_{(2,-1 / 2)}$ contained in the third subspace is related to the eigenvalue $\mu$ then the eigenvector $\left(0, v_{1}, 0,-v_{3} ; 0, v_{5}, 0,-v_{7} ; 0, v_{9}, 0,-v_{11} ; \ldots\right)^{T} \in \ell_{(2,-1 / 2)}$ is related to the eigenvalue $-\mu$. Using that these eigenvectors are orthogonal we have $\sum_{k=0}^{\infty} v_{4 k+1}^{2}=\sum_{k=0}^{\infty} v_{4 k+3}^{2}$. Theorem 16 implies that

- $\frac{1}{2}+\mu$ is an eigenvalue of the Schur complement operator with twice the multiplicity of $\mu$ in (15). The eigenfunctions are $2 \operatorname{Re}\left(\frac{1}{g^{\prime}(z)} \sum_{n=0}^{\infty} v_{2 n+1} z^{2 n+1}\right)$ and $2 \operatorname{Im}\left(\frac{1}{g^{\prime}(z)} \sum_{n=0}^{\infty}(-1)^{n} v_{2 n+1} z^{2 n+1}\right)$,

- $\frac{1}{2}-\mu$ is an eigenvalue of the Schur complement operator with twice the multiplicity of $\mu$ in (15). The eigenfunctions are

$$
2 \operatorname{Im}\left(\frac{1}{g^{\prime}(z)} \sum_{n=0}^{\infty} v_{2 n+1} z^{2 n+1}\right) \text { and } 2 \operatorname{Re}\left(\frac{1}{g^{\prime}(z)} \sum_{n=0}^{\infty}(-1)^{n} v_{2 n+1} z^{2 n+1}\right) .
$$

4. The mapping function of the unit disc onto a regular polygon of order $M$ is

$$
g(z)=\int_{0}^{z} \frac{1}{\left(1-t^{M}\right)^{\frac{2}{M}}} d t=\sum_{n=0}^{\infty} \frac{\Gamma\left(n+\frac{2}{M}\right)}{\Gamma\left(\frac{2}{M}\right)} \frac{z^{n M+1}}{(n M+1) n !} .
$$

The nonzero elements of the infinite matrix $\mathcal{M}_{S}$ are $s_{n M}$ where $n=0,1,2, \ldots$ Therefore, similar to the case of the square, we will have eigenvalues of the Schur complement operator with multiplicity more than 1.

Motivated by the Examples 3 and 4 we can generally formulate the following

THEOREM 19. Let the bijective conformal mapping of the unit disc be of the form

$$
g(z)=\sum_{n=0}^{\infty} a_{n M+1} z^{n M+1}
$$

where $a_{1} \neq 0$ and $M \geq 2$ is an arbitrary integer, then we have eigenvalues of the Schur complement operator with multiplicity more than 1.

Proof. If $g$ is of the form (42) then we have, by multiplying the derivative of (42) and (8),

$$
1=g^{\prime}(z) \cdot \frac{1}{g^{\prime}(z)}=\sum_{n=0}^{\infty}\left\{\sum_{k=0}^{M-1}\left[\sum_{\ell=0}^{n}(\ell M+1) a_{\ell M+1} b_{(n-\ell) M+k}\right] z^{n M+k}\right\} .
$$


This can be written in the infinite block matrix form

$$
\left(\begin{array}{ccccccc}
a_{1} I & 0 & \ldots & & & \\
(M+1) a_{M+1} I & a_{1} I & 0 & \ldots & & \\
\ddots & \ddots & \ddots & \ddots & & \\
(n M+1) a_{n M+1} I & \ldots & \ldots & a_{1} I & 0 & \ldots & \\
\ddots & & & & & \ddots & \ddots
\end{array}\right)\left(\begin{array}{c}
\tilde{b}_{0} \\
\tilde{b}_{1} \\
\vdots \\
\tilde{b}_{n} \\
\vdots
\end{array}\right)=\left(\begin{array}{c}
\vec{e}_{1} \\
0 \\
\vdots \\
0 \\
\vdots
\end{array}\right),
$$

where $I \in \mathbb{R}^{M \times M}$ is the unit matrix, $\vec{e}_{1}=(1,0, \ldots, 0)^{T} \in \mathbb{R}^{M}$ and

$$
\tilde{b}_{n}:=\left(b_{n M}, b_{n M+1}, \ldots, b_{n M+M-1}\right)^{T}
$$

for $n=0,1, \ldots$ There follows $\tilde{b}_{0}=\frac{1}{a_{1}} \vec{e}_{1}$ and further by induction $\tilde{b}_{n}=C \vec{e}_{1}$ where $C \in \mathbb{C}$ is a constant composed of the coefficients of (42). This implies that, in (8), $b_{\ell} \neq 0$ only for $\ell=n M$ where $n=0,1, \ldots$. From the series form of $g(z)$ we see that $a_{k} \neq 0$ only for $k=n M+1$. This means - using the formula in Remark 18 - that $s_{\ell} \neq 0$ only for $\ell=n M$ where $n=0,1, \ldots$

For $k=0,1, \ldots,\left[\frac{M}{2}\right]$, let us introduce the pairwise orthogonal subspaces $L_{k}$ of $\ell_{(2,-1 / 2)}$ as follows. We shall say that $v:=\left(v_{0}, v_{1}, \ldots\right)^{T} \in L_{k}$, if $v \in \ell_{(2,-1 / 2)}$ and if

$$
v_{\ell}=0 \text { for all } \ell \neq n M+k,(n+1) M-k,
$$

where $n=0,1, \ldots$ and $k$ is a fixed integer between 0 and $\left[\frac{M}{2}\right]$. If $v \in L_{k}$ for $k \neq 0, \frac{M}{2}$ $\left(\frac{M}{2}\right.$ only in case $M$ is even) is an eigenvector of $\mathcal{M}_{S} \circ \mathcal{C}$ to the eigenvalue $\mu$ then $\tilde{v}$ is an eigenvector to the eigenvalue $-\mu$ where the elements of $\tilde{v}$ are defined by

$$
\begin{aligned}
\tilde{v}_{n M+k} & =v_{n M+k}, \\
\tilde{v}_{(n+1) M-k} & =-v_{(n+1) M-k}
\end{aligned}
$$

for $n=0,1, \ldots$, otherwise $\tilde{v}_{\ell}=v_{\ell}=0$. Therefore $\frac{1}{2}+\mu$ and $\frac{1}{2}-\mu$ are eigenvalues of the Schur complement with twice the multiplicity of $\mu$ in (15).

REMARK 20. If the mapping function is of the form as in Theorem 19 then

$$
g\left(e^{i k \frac{2 \pi}{M}} z\right)=e^{i k \frac{2 \pi}{M}} g(z), \text { where } k=0,1, \ldots, M-1 .
$$

The left-hand side of this equality means that we first rotate the unit disc by $k \frac{2 \pi}{M}$ and then we map it onto the domain $\Omega$. The right-hand side means that we first map the unit disc onto $\Omega$ and then we rotate $\Omega$ by $k \frac{2 \pi}{M}$. Therefore $\Omega$ is invariant under any rotation by the angle $k \frac{2 \pi}{M}$, consequently $\Omega$ has $M$ symmetry axes. Theorem 19 says that in the case of symmetrical domains the Schur complement operator has multiple eigenvalues depending on the number of the symmetry axes. Moreover each subspace $L_{k}$ of $\ell_{(2,-1 / 2)}$ seems to be connected with one or two symmetry axes.

5. Continuous dependence of $\mathcal{M}_{S}$ on the domain. We investigate the dependence of $\mathcal{M}_{S}$ on the domain $\Omega$ onto which the unit disc $D$ is mapped. Let be $\tilde{g}(z)=\eta(g(z))$ where the function $\eta$ is the univalent conformal mapping of the domain $\Omega$ onto the domain $\tilde{\Omega}$. So the univalent conformal mapping $\tilde{g}=\eta \circ g$ maps the unit disc $D$ onto $\tilde{\Omega}$. Using the notation $w=g(z)$, there follows

$$
\tilde{g}^{\prime}(z)=\eta^{\prime}(w) \cdot g^{\prime}(z) \text {. }
$$


We have from (21) for the entries of the matrix $\mathcal{M}_{S}$ and $\tilde{\mathcal{M}}_{S}$ :

$$
\begin{aligned}
& s_{k}=\frac{1}{\pi} \int_{D} \bar{z}^{k} \frac{g^{\prime}(z)}{\overline{g^{\prime}(z)}} d x d y, \\
& \tilde{s}_{k}=\frac{1}{\pi} \int_{D} \bar{z}^{k} \frac{\tilde{g}^{\prime}(z)}{\tilde{g}^{\prime}(z)} d x d y .
\end{aligned}
$$

Subtract (44) and (45):

$$
\tilde{s}_{k}-s_{k}=\frac{1}{\pi} \int_{|z|<1} \bar{z}^{k}\left(\frac{\tilde{g}^{\prime}(z)}{\overline{\tilde{g}^{\prime}(z)}}-\frac{g^{\prime}(z)}{\overline{g^{\prime}(z)}}\right) d x d y .
$$

Using (43), we obtain

$$
\frac{\tilde{g}^{\prime}(z)}{\overline{\tilde{g}^{\prime}(z)}}-\frac{g^{\prime}(z)}{\overline{g^{\prime}(z)}}=\left(\frac{\eta^{\prime}(w)}{\overline{\eta^{\prime}(w)}}-1\right) \frac{g^{\prime}(z)}{\overline{g^{\prime}(z)}}=\left(e^{2 i \arg \eta^{\prime}(w)}-1\right) \frac{g^{\prime}(z)}{\overline{g^{\prime}(z)}} .
$$

The identity

$$
e^{2 i \arg \eta^{\prime}(w)}-1=2 i e^{i \arg \eta^{\prime}(w)} \sin \left(\arg \eta^{\prime}(w)\right),
$$

gives

$$
\left|\frac{\tilde{g}^{\prime}(z)}{\overline{\tilde{g}^{\prime}(z)}}-\frac{g^{\prime}(z)}{\overline{g^{\prime}(z)}}\right| \leq 2\left|\sin \left(\arg \eta^{\prime}(w)\right)\right|=2\left|\sin \left(\arg \tilde{g}^{\prime}(z)-\arg g^{\prime}(z)\right)\right| .
$$

Estimating the integral (46), we finally have

$$
\left|\tilde{s}_{k}-s_{k}\right| \leq 4 \max _{|z| \leq 1}\left|\sin \left(\arg \tilde{g}^{\prime}(z)-\arg g^{\prime}(z)\right)\right| .
$$

We further examine the following eigenvalue problem: find $\mu \in \mathbb{C}$ and the convergent series $p(z)=\sum_{m=0}^{\infty} p_{m} z^{m}$ such that

$$
\int_{D} \overline{p(z) q(z)} \frac{g^{\prime}(z)}{\overline{g^{\prime}(z)}} d x d y=-2 \mu \int_{D} p(z) \overline{q(z)} d x d y
$$

holds for all convergent series $q(z)=\sum_{m=0}^{\infty} q_{m} z^{m}$. This problem is in fact (22) for convergent power series. There is a similar eigenvalue problem for $\tilde{g}=\eta \circ g$ :

$$
\int_{D} \overline{\tilde{p}(z) q(z)} \frac{\tilde{g}^{\prime}(z)}{\tilde{g}^{\prime}(z)} d x d y=-2 \tilde{\mu} \int_{D} \tilde{p}(z) \overline{q(z)} d x d y .
$$

Substitute $q=\tilde{p}$ into (49) and $q=p$ into (50), and subtract the equations:

$$
\int_{D} \overline{\tilde{p}(z) p(z)}\left(\frac{\tilde{g}^{\prime}(z)}{\tilde{\tilde{g}}^{\prime}(z)}-\frac{g^{\prime}(z)}{\overline{g^{\prime}(z)}}\right) d x d y=\int_{D}(2 \mu \overline{\tilde{p}(z) \overline{p(z)}}-2 \tilde{\mu} \tilde{p}(z) \overline{p(z)}) d x d y .
$$

Using $|b||| \tilde{a}|-| a|| \leq|b|\left|\tilde{a} \frac{\bar{b}}{b}-a\right|=|\tilde{a} \bar{b}-a b|$ for $\tilde{a}, a, b \in \mathbb{C}, b \neq 0$, we estimate the right-hand side:

$$
2|| \tilde{\mu}|-| \mu|| \cdot\left|\int_{D} \tilde{p}(z) \overline{p(z)} d x d y\right| \leq\left|\int_{D}(2 \mu \overline{\tilde{p}(z) \overline{p(z)}}-2 \tilde{\mu} \tilde{p}(z) \overline{p(z)}) d x d y\right| .
$$


We estimate the left-hand side

$$
\left|\int_{D} \overline{\tilde{p}(z) p(z)}\left(\frac{\tilde{g}^{\prime}(z)}{\overline{\tilde{g}^{\prime}(z)}}-\frac{g^{\prime}(z)}{\overline{g^{\prime}(z)}}\right) d x d y\right| \leq \max _{z \in D}\left|\frac{\tilde{g}^{\prime}(z)}{\overline{\tilde{g}^{\prime}(z)}}-\frac{g^{\prime}(z)}{\overline{g^{\prime}(z)}}\right| \int_{D}|\tilde{p}(z) p(z)| d x d y .
$$

Using (43) and the identity (47), there follows

$$
\| \tilde{\mu}|-| \mu|| \frac{\left|\int_{D} \tilde{p}(z) \overline{p(z)} d x d y\right|}{\int_{D}|\tilde{p}(z) p(z)| d x d y} \leq \max _{z \in D}\left|\sin \left(\arg \tilde{g}^{\prime}(z)-\arg g^{\prime}(z)\right)\right| .
$$

Hence, we have proved the following result.

Corollary 21. Let $g$ be a bijective conformal mapping of the unit disc D onto the simply-connected plane domain $\Omega$. Let $\tilde{g}$ be defined by $\tilde{g}(z)=\eta(g(z))$, where the function $\eta$ is the univalent conformal mapping of the domain $\Omega$ onto the domain $\tilde{\Omega}$. We have the stability estimates (48) for the entries of $\mathcal{M}_{S}$, and (51) for the eigenvalues of (15).

REMARK 22 . We suppose that $\Omega$ is an open, bounded, simply connected domain. Let the boundary $\partial \Omega$ be twice continuously differentiable so that it admits a $C^{2}$ parametrization $w(t), 0 \leq t \leq T$. Denote the unit tangent to $\partial \Omega$ at $w$ by $\dot{\gamma}(w)=\frac{\dot{w}(t)}{|\dot{w}(t)|}$. Let $R(w)$ denote the conformal mapping $\bar{\Omega}$ onto the closed unit disc. We have for $w \in \partial \Omega($ see $[12])$

$$
R(w)=-i \dot{\gamma}(w) \frac{R^{\prime}(w)}{\left|R^{\prime}(w)\right|}
$$

Because $R(w)$ is the inverse of $g(z)$, there follows $R^{\prime}(w)=\frac{1}{g^{\prime}(z)}$, and using (52), we have

$$
e^{i \arg g^{\prime}(z)}=\frac{-i}{z} \dot{\gamma}(w)
$$

for $z \in \partial D$ and for the corresponding $w=g(z) \in \partial \Omega$. From this we obtain

$$
e^{i \arg \tilde{g}^{\prime}(z)}-e^{i \arg g^{\prime}(z)}=\frac{-i}{z}(\dot{\tilde{\gamma}}(\tilde{w})-\dot{\gamma}(w))
$$

for $z \in \partial D, w \in \partial \Omega, \tilde{w} \in \partial \tilde{\Omega}$, where $\tilde{w}=\tilde{g}(z)=\eta(g(z))$. Using the identity

$$
e^{i \arg \tilde{g}^{\prime}(z)}-e^{i \arg g^{\prime}(z)}=2 i \sin \frac{\arg \tilde{g}^{\prime}(z)-\arg g^{\prime}(z)}{2} e^{\frac{1}{2}\left(\arg \tilde{g}^{\prime}(z)+i \arg g^{\prime}(z)\right)}
$$

and taking the absolute value, there follows for $z \in \partial D, w \in \partial \Omega, \tilde{w} \in \partial \tilde{\Omega}$ :

$$
|\dot{\tilde{\gamma}}(\tilde{w})-\dot{\gamma}(w)|=2\left|\sin \frac{\arg \tilde{g}^{\prime}(z)-\arg g^{\prime}(z)}{2}\right| \leq\left|\arg \tilde{g}^{\prime}(z)-\arg g^{\prime}(z)\right| .
$$

We finally have the estimate

$$
|\dot{\tilde{\gamma}}(\tilde{w})-\dot{\gamma}(w)| \leq \max _{|z| \leq 1}\left|\arg \tilde{g}^{\prime}(z)-\arg g^{\prime}(z)\right|
$$


for all $w \in \partial \Omega$ and $\tilde{w} \in \partial \tilde{\Omega}$ corresponding to each other by $\tilde{w}=\eta(w)$. This shows together with Corollary 21 that for domains with $C^{2}$-boundary the value

$$
\max _{|z| \leq 1}\left|\arg \tilde{g}^{\prime}(z)-\arg g^{\prime}(z)\right|
$$

is a common upper bound in (53) and in (48), (51), however with other constants.

In the following let be $\tilde{g}(z)=g(z)+\varepsilon(z)$ with $\varepsilon(z)=\sum_{m=0}^{\infty} \varepsilon_{m} z^{m}$. Using the identity $\frac{a}{b}-\frac{c}{d}=\frac{a-c}{d}-\frac{a}{b} \frac{b-d}{d}$ we have

$$
\left|\frac{\tilde{g}^{\prime}(z)}{\overline{\tilde{g}^{\prime}(z)}}-\frac{g^{\prime}(z)}{\overline{g^{\prime}(z)}}\right|=\left|\frac{\tilde{g}^{\prime}(z)-g^{\prime}(z)}{\overline{g^{\prime}(z)}}-\frac{\tilde{g}^{\prime}(z)}{\overline{\tilde{g}^{\prime}(z)}} \cdot \frac{\tilde{g}^{\prime}(z)-g^{\prime}(z)}{\overline{g^{\prime}(z)}}\right| \leq 2\left|\frac{\varepsilon^{\prime}(z)}{g^{\prime}(z)}\right| .
$$

We can estimate the difference of (44) and (45):

$$
\left|\tilde{s}_{k}-s_{k}\right| \leq 4 \max _{|z| \leq 1}\left|\frac{\varepsilon^{\prime}(z)}{g^{\prime}(z)}\right| .
$$

This shows the continuous dependence of the matrix $\mathcal{M}_{S}$ on $\varepsilon^{\prime}(z)$ and means also the continuous dependence of the eigenvalues of (15) in case $g$ and $\varepsilon$ are polynomials. If they are not polynomials then we can estimate as before. After substituting $q=\tilde{p}$ into (49) and $q=p$ into (50), we subtract the resulting equations. There follows

$$
\| \tilde{\mu}|-| \mu|| \frac{\left|\int_{D} \tilde{p}(z) \overline{p(z)} d x d y\right|}{\int_{D}|\tilde{p}(z) p(z)| d x d y} \leq \max _{z \in D}\left|\frac{\varepsilon^{\prime}(z)}{g^{\prime}(z)}\right|
$$

Remark 23. $\tilde{g}(z)=g(z)+\varepsilon(z)$ will not be necessarily univalent. However the above estimates can be useful if we intend to approximate $g(z)$ by a polynomial $\tilde{g}(z)$ cutting off the remainder term $\varepsilon(z)$. So we have the possibility to calculate numerically some approximate eigenvalues.

REMARK 24. Let $g_{M}(z)=z-\frac{c}{M} z^{M}$ for $M=1,2, \ldots$ be the mapping of the unit disc $\mathrm{D}$ onto a domain $\Omega_{M}$, investigated in the Example 2 in Section 3. Let further be $g(z)=z$ the mapping of the unit disc onto itself. We compute

$$
\begin{aligned}
\left\|g_{M}-g\right\|_{0}^{2} & =\frac{|c|^{2}}{M^{2}} \int_{D} z^{M} \bar{z}^{M} d x d y=\frac{\pi|c|^{2}}{M^{2}(M+1)}, \\
\max _{z \in D}\left|g_{M}(z)-g(z)\right| & =\frac{|c|}{M} \max _{z \in D}\left|z^{M}\right|=\frac{|c|}{M} \\
\left\|g_{M}^{\prime}-g^{\prime}\right\|_{0}^{2} & =\frac{\pi|c|^{2}}{M}, \\
\max _{z \in D}\left|g_{M}^{\prime}(z)-g^{\prime}(z)\right| & =|c| \max _{z \in D}|z|^{M-1}=|c| .
\end{aligned}
$$

These equalities show $\lim _{M \rightarrow \infty} g_{M}=g$ in the $L_{2}$ and maximum norm on $D$, further $\lim _{M \rightarrow \infty} g_{M}^{\prime}=g^{\prime}$ is valid in the $L_{2}$ norm (but not in the maximum norm). In this sense we have a sequence of domains $\Omega_{M}, M=1,2, \ldots$ converging to the unit disc $D$. The limit of the inf-sup constants (32) of the domains is however

$$
\lim _{M \rightarrow \infty} \beta_{0}^{2}\left(\Omega_{M}\right)=\frac{1}{2}-\frac{|c|}{4}<\frac{1}{2}=\beta_{0}^{2}(D) .
$$


If we slightly change the mapping polynomial of Example 2 in Section 3, i.e. we investigate $\hat{g}_{M}: D \rightarrow \hat{\Omega}_{M}$,

$$
\hat{g}(z)=z-\frac{c}{M^{2}} z^{M},
$$

which is also univalent for $c \in \mathbb{R},|c| \leq 1$, then we get

$$
\beta_{0}^{2}\left(\hat{\Omega}_{M}\right)= \begin{cases}\frac{1}{2}-\frac{|c|}{4 M}\left(1+\frac{1}{M}\right) & \text { for } M \text { odd } \\ \frac{1}{2}-\frac{|c|}{4 M} \sqrt{1+\frac{2}{M}} & \text { for } M \text { even. }\end{cases}
$$

We obtain in this modified case $\hat{g}_{M} \rightarrow g$ and $\hat{g}_{M}^{\prime} \rightarrow g^{\prime}$ for $M \rightarrow \infty$ in both $\left(L_{2}\right.$ and maximum) norms. We also have $\lim _{M \rightarrow \infty} \beta_{0}^{2}\left(\hat{\Omega}_{M}\right)=\beta_{0}^{2}(D)$.

As one sees, our polynomial examples may seem far from practical relevance. But they clearify important questions: the above examples show the fact that neither the convergence of the mapping function nor the convergence of its derivative in the $L_{2}$ norm are sufficient to the convergence of the inf-sup constants of the domains to that of the limit domain.

\section{REFERENCES}

[1] Aristov P.P. And Chizhonkov E.V., On the constant in the LBB condition for rectangular domains, Report No. 9534, Dept. Math., Univ. of Nijmegen, Sept. 1995.

[2] Arushanyan I.O. and Chizhonkov E.V., Numerical investigation of the constant in the infsup inequality using boundary integral equations. In: Packages of Programmes for Applications, V.A. Morozov, O.B. Arushanyan (eds.), Editing House of Moscow Univ., 1997, pp. $49-59$.

[3] Chizhonkov E.V., On the constant in the LBB condition for ring domains, Report No. 9537, Dept. Math., Univ. of Nijmegen, Sept. 1995.

[4] Chizhonkov E.G., On spectral properties of the pressure operatore induced by the Stokes equations, In: Proceedings of the Conference in memory of the 175th birthday of P.L. Chebyshev, v. 2. Editing House of the Mech.-Math. Faculty of Moscow Univ., 1996, pp. 363-366.

[5] Chizhonkov E.V. And Olshanski M.A., On the domain geometry dependence of the LBB condition, Math. Modell. Numer. Analysis, 34:5 (2000), pp. 935-951.

[6] Costabel M. And Dauge M., On the Cosserat spectrum in polygons and polyhedra, IRMAR Conference Lausanne 2000.

[7] Crouzeix M., Étude d'une méthode de linéarisation. Résolution numérique des équations Stokes stationnaires, In: Cahier de l'INRIA 12, 1974, pp. 139-244.

[8] Crouzeix M., On an operator related to the convergence of Uzawa's algorithm for the Stokes equation, in: Computational Science for the 21st Century (J. Périaux et al., eds.). New York: Wiley 1997, pp. 242-249.

[9] Friedrichs K., On certain inequalities and characteristic value problems for analytic functions and for functions of two variables, Trans. AMS, 41 (1937), pp. 321-364.

[10] Horgan C.O. and Payne L.E., On inequalities of Korn, Friedrichs and Babuška-Aziz, Achive Rat. Mech. Anal., 82 (1983), pp. 165-179.

[11] Kratz W. and Peyerimhoff A., A numerical algorithm for the Stokes problem based on an integral equation for the pressure via conformal mappings, Numerische Mathematik, 58 (1990), pp. 255-272.

[12] Lee B. And Trummer M.R., Multigrid conformal mapping via the Szegö kernel, Electronic Transactions on Numerical Analysis, 2 (1994), pp. 22-43.

[13] Maday Y., Meiron D., Patera A.T., Ronquist E.M., Analysis of iterative methods for the steady and unsteady Stokes problem: application to spectral element discretizations, SIAM J. Sci. Comput., 14 (1993), pp. 310-337.

[14] Mikhlin S.G., The spectrum of the operator pencil of elasticity theory, Uspekhi Mat. Nauk 28, No. 3 (171), (1973), pp. 43-82.

[15] Nehari Z., Conformal Mapping, McGraw-Hill Book Company, Inc., New York, 1952.

[16] Stoyan G., Towards discrete Velte decompositions and narrow bounds for inf-sup constants, Computers \& Maths. with Appls., 38, 7-8, (1999), pp. 243-261. 
[17] Stoyan G., Iterative Stokes solvers in the harmonic Velte subspace, Computing, 67 (2000), pp. $13-33$.

[18] Velte W., On optimal constants in some inequalities, in: The Navier-Stokes Equations, Theory and Numerical Methods (Heywood J.G. et al., eds.). Lecture Notes in Math. 1431. SpringerVerlag Berlin 1990, pp. 158-168.

[19] Velte W., On inequalities of Friedrichs and Babuška-Aziz, Meccanica, 31 (1996), pp. 589-596.

[20] Zimmer S., Rand-Druckkorrektur für die Stokes-Gleichung, Thesis, Techn. Univ. München, 1996. 